\title{
Pharmacokinetic Parameters Domain
}

National Cancer Institute

\section{Source}

National Cancer Institute. Pharmacokinetic Parameters Domain. NCI Thesaurus. Code C49607.

A subject domain utilized for the submission of information encompassing and representing data, vocabulary or records related to pharmacokientic parameters. 